\title{
Réalité virtuelle et jeux: de nouveaux outils pour des apprentissages plurilingues?
}

Gérald Schlemminger, Mickaël Roy, Manuel Veit, Antonio Capobianco et Gilles Noeppel

\section{(2) OpenEdition \\ Journals}

Édition électronique

URL : http://journals.openedition.org/esp/2731

DOI : $10.4000 /$ esp.2731

ISSN : 2532-0319

Éditeur

Centre d'Information sur l'Éducation Bilingue et Plurilingue

\section{Édition imprimée}

Date de publication : 1 décembre 2013

Pagination : $29-42$

ISSN : 1127-266X

\section{Référence électronique}

Gérald Schlemminger, Mickaël Roy, Manuel Veit, Antonio Capobianco et Gilles Noeppel, « Réalité virtuelle et jeux: de nouveaux outils pour des apprentissages plurilingues ? », Éducation et sociétés plurilingues [En ligne], 35 | 2013, mis en ligne le 01 février 2020, consulté le 17 février 2021. URL: http://journals.openedition.org/esp/2731 ; DOI : https://doi.org/10.4000/esp.2731 


\title{
RÉALITÉ VIRTUELLE ET JEUX: DE NOUVEAUX OUTILS POUR DES APPRENTISSAGES PLURILINGUES?
}

\author{
Gérald Schlemminger, Mickaël Roy, \\ Manuel VeIt, Antonio GaPobianco, Gilles NoEPPEL
}

Der Artikel erörtert die Verwendung der virtuellen Realität zur Förderung der Mehrsprachigkeit. Diese neue Technologie macht es möglich, dass der Lernende durch die Integration von sensorischen und haptischen Parametern sowie Gestenerkennung in das vom Computer generierte Umfeld eintauchen kann und somit die subjektive, gefuihlsmäßige Erfahrung des Spielenden erhöht wird. Durch diese Wahrnehmungsillusion entsteht beim Lernenden ein Präsenzgefihl in der virtuellen Umgebung, welches das Erlernen von Lerninhalten begünstigt. Um eine dem Lernenden angepasste kognitive Förderung sowie eine pragmatische und kontextualisierte Anwendung der Fremdsprache zu gewährleisten, stellt das Serious Game eine Anwendungsmöglichkeit der virtuellen Realität dar.

The article examines the use of virtual reality as a tool for multilingualism. This new technology allows for sensory immersion and the learner's gestural involvement in a computer-generated environment. It is through this perceptual illusion that the learner develops a feeling of presence in the virtual environment, thereby promoting both language and additional subject learning. The use of serious games is a path through which virtual reality may be used as a learning tool for multilingualism, as it offers a pragmatic and contextualized use of the language and its cognitive demands can be adapted to the individual learner.

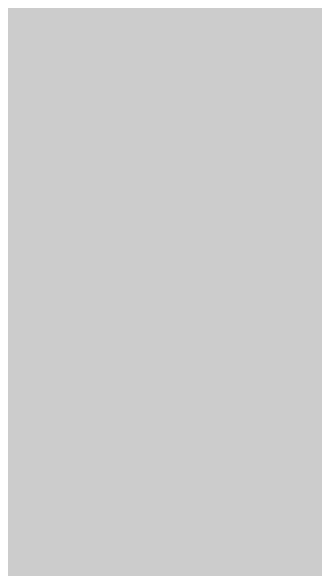

$\mathrm{D}$ ans cet article, nous chercherons à savoir dans quelle mesure les nouveaux outils de la technologie de l'information et de la communication (TIC), tels que la réalité virtuelle 3D et les jeux vidéo, peuvent être utiles pour promouvoir le plurilinguisme dans l'enseignement des langues. Des applications de la réalité virtuelle sont largement utilisées dans des domaines tels que l'aviation (simulateur de vol), la chirurgie, la mécanique (réparation assistée des moteurs), la prévention routière ou les jeux vidéo. Dans l'éducation et pour les apprentissages scolaires, ces outils ne sont pas encore très répandus et les expériences ne sont que rarement publiées.

Certes, il existe des exemples: Pierre Wild, professeur de sciences physiques dans un lycée alsacien, a expérimenté des outils pédagogiques basés sur l'utilisation d'OpenSim (1). Il a réalisé un espace de simulation en ligne, réservé à un usage pédagogique, où ses élèves de seconde, présents dans le monde virtuel via leur avatar, réalisent des expériences scientifiques dans le cadre du module de chimie 
Réalité virtuelle et jeux: de nouveaux outils pour des apprentissages plurilingues?

G. SCHLEMmingeR, M. RoY, M.Veit, A. Capobianco,

G. NOEPPEL

DE QUELLES

TECHNOLOGIES PARLONS-NOUS?

\section{LA RÉALITÉ VIRTUELLE: IMMERSION, INTERACTION ET PRÉSENCE}

«sciences et laboratoire» (Blocci 2012).

Pour l'apprentissage des langues, l'université populaire de Goslar (VHS Goslar) propose sur la plateforme «Second Life» un espace appelé «ohs-Insel). Elle y offre des cours de langues (payants) mais aussi un espace de discussion. Représentés par leur avatar, les participants y échangent à l'écrit ou à l'oral. Le Goethe-Institut dispose de son «île» appelée «Deutschlern-Treff», où les participants peuvent converser gratuitement en allemand avec des enseignants et d'autres participants.

Mais avant d'approfondir le sujet, nous proposons de faire un tour d'horizon des questions technologiques. Ensuite, nous présenterons le projet EVEIL-3D qui étudie plus particulièrement l'impact de ces technologies sur l'apprentissage guidé des langues.

Nous aborderons d'abord la notion de «réalité virtuelle» avant de définir les «jeux sérieux». Ensuite, nous les mettrons en rapport avec l'enseignement des langues et ce que ces nouvelles technologies peuvent lui apporter.

Nous aborderons les différents aspects de la réalité virtuelle ainsi que ses concepts essentiels, tels que la notion d'interaction homme-machine, d'immersion et de présence.

Fuchs et al. (2006: 5) définissent la réalité virtuelle de la manière suivante: «La finalité de la réalité virtuelle est de permettre à une personne (ou à plusieurs) de déployer une activité sensori-motrice et cognitive dans un monde artificiel, créé numériquement, qui peut être imaginaire, symbolique, ou une simulation de certains aspects du monde réel».

Cette définition permet de mettre en avant un aspect fondamental de la réalité virtuelle: la personne interagit physiquement avec des éléments virtuels. Mais ne succombons pas à l'erreur fréquente de croire que la finalité de la réalité virtuelle consiste en la création d'un monde aussi réaliste que possible.

Ce monde virtuel peut être imaginaire; il ne constitue plus une simulation du monde réel et de ses conditions de vie. Il peut s'agir de créer un monde symbolique qui permet à l'utilisateur d'obtenir une représentation mentale améliorée de son environnement, par exemple le flux acoustique de l'oreille interne pour un cours de biologie.

Des signaux de tout ordre peuvent être ajoutés à un environnement virtuel réaliste pour attirer par exemple l'attention sur un danger potentiel. Avec la réalité augmentée, on visualisera la réalité environnementale à laquelle le concepteur aura associé numériquement des objets, des phénomènes physiques, visibles par exemple à l'aide d'une paire de lunettes spécifiques. La figure 1 montre, sur un axe horizon- 
Réalité virtuelle et jeux: de nouveaux outils pour des apprentissages plurilingues?

G. SChlemmingeR, M. Roy, M. Veit, A. Capobianco,

G. NOEPPEL tal, le continuum allant de l'environnement réel (ou écologique) à un monde entièrement virtuel, en passant par la réalité augmentée et la virtualité augmentée (monde virtuel avec des objets du réel), désignées comme «réalité mixte».

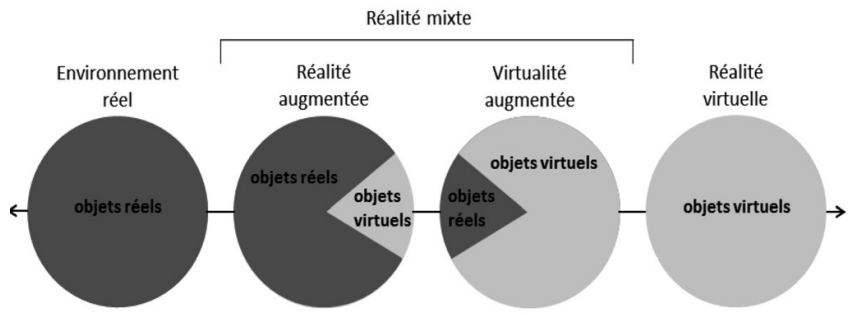

Fig. : Représentation simplifiée du continuum réalité-virtualité (d'après P. Milgram, F. Kishino 1994)

«L'utilisateur agit sur l'environnement virtuel grâce à l'usage d'interfaces motrices qui captent ses actions (gestes, déplacements, voix, etc.). Ces activités sont transmises au calculateur [ordinateur] qui linterprète comme une demande de modification de l'environnement. Conformément à cette sollicitation de modification, le calculateur évalue les transformations à apporter à l'environnement virtuel et les restitutions sensorielles (images, son, efforts, etc.) à transmettre aux interfaces sensorielles. Cette boucle en environnement virtuel interactif n'est que la transposition de la boucle 'perception, cognition, action' du comportement de l'homme dans un mode réel». (Fuchs et al: 9 ).

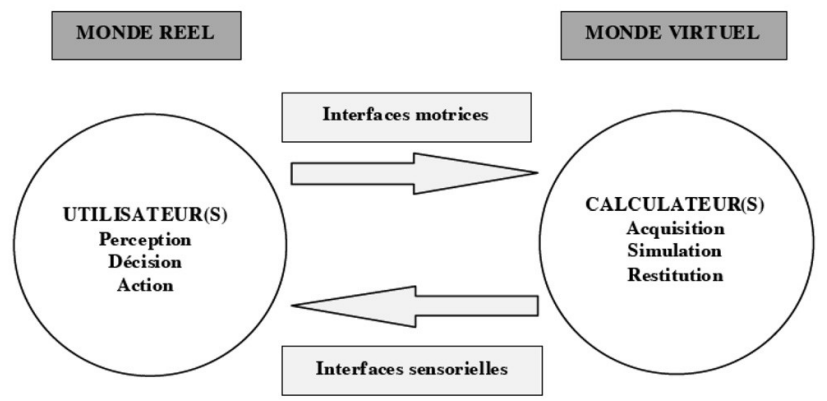

Fig. 2: La boucle «perception, cognition, action» passant par le monde virtuel (de Fuchs, et al. 2006: 9) 
Réalité virtuelle et jeux: de nouveaux outils pour des apprentissages plurilingues?

G. SChlemminger, M. Roy, M. Veit, A. Capobianco,

G. NOEPPEL
Mais les mêmes auteurs avertissent également de deux phénomènes pouvant perturber cette boucle: la latence et les incohérences sensorimotrices. La latence est le décalage entre une action de l'utilisateur et la perception par celui-ci des modifications de l'environnement virtuel engendrées par son action (visuelles, sonores...). Si l'écart entre l'action et ses répercussions sur l'environnement est trop important, l'environnement virtuel perd en crédibilité. Rappelons avec Bouvier (2008: 12) l'importance de la crédibilité de l'expérience pour l'utilisateur: l'immersion sensorielle et l'interaction doivent lui permettre de se prendre au jeu et ressentir un sentiment de présence dans l'environnement virtuel.

D'après Slater (2003: 3), l'immersion se définit comme le niveau de fidélité sensorielle du stimulus de réalité virtuelle. En d'autres termes, l'immersion dépend de la technologie utilisée pour générer l'environnement, et non de l'expérience subjective vécue par l'utilisateur. On pourra considérer qu'un environnement sera plus ou moins immersif selon que la technologie utilisée permet d'obtenir un champ de vision plus ou moins large, un réalisme graphique plus ou moins important, une richesse sensorielle (par exemple, par le biais de dispositifs tactiles ou de stéréoscopie) plus ou moins importante.

L'immersion favorise la présence. À l'inverse de l'immersion, la présence renvoie au vécu subjectif d'un individu face à l'environnement immersif. Elle est associée à la propension d'un individu à répondre à l'environnement virtuel comme si celui-ci était réel (Sanchez-Vives \& Slater 2005). Elle est donc en lien avec la perception et la réponse émotionnelle de l'individu, et caractérise son sentiment d'être effectivement présent dans le monde virtuel.

Il y a bien sûr des interactions entre les concepts de présence et d'immersion. Les facteurs pouvant avoir une influence sur la présence sont nombreux; des recherches se sont penchées sur l'analyse de l'influence de quelques paramètres liés à l'immersion:

Affichage: la fréquence d'affichage, la stéréoscopie, le suivi des mouvements de la tête et la gestion du champ visuel sont des facteurs qui semblent tous être positivement corrélés à la présence.

Réalisme visuel: la qualité des graphismes ne semble pas être un facteur primordial pour le sentiment de présence.

Environnement sonore: la présence de sons dans l'environnement à un effet positif sur le sentiment de présence.

Retour haptique: de la même façon, la présence d'un retour tactile, c'est-à-dire d'un dispositif (gants de données par exemple) permettant à l'utilisateur d'avoir la sensation physique de toucher les objets virtuels, semble avoir un effet positif sur la présence. 
Réalité virtuelle et jeux: de nouveaux outils pour des apprentissages plurilingues?

G. SChlemminger, M. Roy, M. Veit, A. Capobianco,

G. NOEPPEL

\section{IMMERSION ET} APPRENTISSAGE

PRÉSENCE ET APPRENTISSAGE
Représentation virtuelle du corps: lors de l'utilisation d'un casque de réalité virtuelle, offrir une modélisation virtuelle du corps de l'utilisateur (par exemple de sa main) semble accroître le sentiment de présence.

Engagement corporel: cette notion fait référence au fait d'inciter l'utilisateur à réaliser les gestes réels de l'action qu'il entreprend dans l'environnement virtuel (par exemple, marcher réellement pour se déplacer dans l'environnement plutôt qu'utiliser un périphérique de type flystick (manette de commande dans un environnement de réalité virtuelle).

De nombreux environnements d'apprentissage en réalité virtuelle ont été développés en vue d'exploiter leurs potentialités de visualisation accrue (notamment les informations liées à la perception spatiale: stéréoscopie, parallaxe (effet du changement de position de l'observateur sur ce qu'il perçoit, etc.). Ils visent en général à favoriser l'apprentissage de gestes et de comportements par la mise en situation et le transfert de compétences du virtuel vers le réel (Patel et al. 2006). Parfois ils servent l'apprentissage de concepts abstraits, par exemple de principes mathématiques ou de principes physiques.

Si les études réalisées tendent à montrer une plus-value liée à l'usage de ces environnements, l'influence effective de l'immersion offerte a été peu étudiée jusqu'à présent. Les seules études approfondies, à notre connaissance, sont celles réalisées par Ragan et al. (2010). Leurs études montrent que le niveau d'immersion a une influence significative sur l'apprentissage, une plus grande immersion entraînant un meilleur apprentissage de contenus disciplinaires, avec par exemple chez Heers (2005), en chimie, une meilleure compréhension des processus.

Il y a néanmoins peu de travaux portant sur l'usage d'environnements immersifs pour l'apprentissage des langues. Dernièrement, les travaux se sont plutôt tournés vers les univers virtuels en ligne, tels que «Second Life», pour proposer des outils d'apprentissage de type «Serious games» (Amoia et al. 2011). Ce type d'environnement escompte tirer parti de l'immersion dans l'environnement pour favoriser l'apprentissage. Ici, le terme immersion renvoie à la notion d'immersion langagière, c'est-àdire le fait de placer l'apprenant dans une situation où il n'a pas d'autre recours que l'utilisation de la langue seconde pour comprendre et communiquer avec son environnement. Cette immersion langagière se différencie de l'immersion sensorielle en réalité virtuelle, plus holistique.

À notre connaissance il existe peu d'études sur l'influence du facteur «présence» sur l'apprentissage. Dans la communauté des sciences de l'éducation, ce concept est décliné sous trois formes (McKerlich et al. 2011): 
Réalité virtuelle et jeux: de nouveaux outils pour des apprentissages plurilingues?

G. SChlemminger, M. Roy, M. Veit, A. Capobianco,

G. NOEPPEL

LEJEU SÉRIEUX: UNE PREMIÈRE APPROGHE
Présence sociale: l'intensité de la projection de l'apprenant dans l'environnement et la perception que d'autres participants peuvent en avoir, dans le cas d'un apprentissage en environnement collaboratif.

Présence cognitive: la capacité de l'apprenant à tirer du sens et renforcer une connaissance par le biais de l'expérience virtuelle.

Présence enseignante: l'influence directe (intervention) ou indirecte (structuration de l'activité pédagogique, scénarisation) de l'enseignant sur l'expérience vécue.

L'expérience éducative surviendrait lorsque l'expérience proposée se place à l'intersection de ces trois facteurs. Une plus grande présence permettrait un apprentissage plus rapide (Lane et al. 2010). L'expérience de P. Wild en sciences physiques illustre bien les différents aspects de la réalité virtuelle et de sa scénarisation pédagogique possible:

«En fait, j'ai reconstitué une scène de crime dans l'univers virtuel. Les élèves devront visiter ce lieu et y trouver des indices. L'objectif sera de faire des expériences réelles en laboratoire pour vérifier les hypothèses suscitées par ces indices. Exemple: des traces de pas contiennent du pollen, quel est l'arbre qui l'a produit? (biologie); un message codé est affiché sur l'écran de l'ordinateur, qu'est-ce qu'il signifie? (cryptographie, mathématiques); une pierre a dû être lancée par la fenêtre, quelle a été sa trajectoire? (physique). Chaque piste étudiée correspond à un élément du programme scolaire de la classe concernée» (interview in Blocci 2012 s.p.).

Cet exemple nous amène à nous intéresser de plus près aux jeux sérieux, en lien avec la scénarisation d'une séquence pédagogique.

L'expression «jeu sérieux», à laquelle est d'ailleurs préférée «serious game», désigne d'après Alvarez (2007):

«[une] application informatique, dont l'intention initiale est de combiner à la fois des aspects sérieux (Serious) tels, de manière non exhaustive, l'enseignement, l'apprentissage, la communication, ou encore l'information, avec des ressorts ludiques issus du jeu vidéo (Game). Une telle association, qui s'opère par l'implémentation [la mise en oeuvre] d'un «scénario pédagogique», a donc pour but de s'écarter du simple divertissement.»

Les serious games sont habituellement classés en cinq catégories:

les serious games engagés (militant games),

les serious games publicitaires (advergames),

les serious games éducatifs (edugames),

les serious games informatifs (informative games) et

les serious games d'entrainement / simulation (training games).

Selon Alvarez (2007), le gameplay semble être le lieu de mise en relation du jeu vidéo avec l'apprentissage. Il décompose le mot pour en expliquer le sens: le «game» représente les règles définissant l'objectif à 
Réalité virtuelle et jeux: de nouveaux outils pour des apprentissages plurilingues?

G. SChlemminger, M. Roy, M. Veit, A. Capobianco,

G. NOEPPEL
JEUX SÉRIEUX POUR L'APPRENTISSAGE DES LANGUES atteindre par le joueur, le «play» spécifie les moyens et les contraintes pour que le joueur atteigne cet objectif. Ces précisions permettent de distinguer différents dispositifs en fonction de la façon dont le «game»» est appréhendé, dont:

Le serious game: les objectifs apparents du jeu correspondent à des objectifs pédagogiques sous-jacents prévus lors de la conception du jeu; les jeux sont conçus dans un objectif d'apprentissage.

Le serious gaming: il s'agit de jeux originellement ludiques détournés dans un objectif d'apprentissage.

Djaouti (2011: 28) va plus loin en proposant une classification qui nous semble pertinente, puisqu'elle spécifie, en plus de ce que permet de faire le jeu sérieux, le domaine d'application (militaire, santé, écologie, mathématiques, etc.) et le public visé, proposant ainsi le modèle G-P-S avec trois critères: le critère «Gameplay » pour la dimension ludique, le critère «permet de» ou «Purpose» pour la finalité sérieuse et le critère «secteur» ou «Scope», prenant en compte le marché et le type de public que le concepteur veut atteindre.

Les jeux numériques en général, comme aide à l'apprentissage des langues, font aujourd'hui partie de l'offre de base des manuels scolaires et de leurs supports en ligne. En didactique des langues, nous parlons de Game-Based Learning, l'apprentissage basé sur des jeux sérieux.

Puisque le jeu peut être considéré comme support d'apprentissage, le pédagogue et le didacticien des langues se doivent d'interroger son utilisation pour l'appropriation d'une langue étrangère. S'intéressant aux performances des apprenants en langue utilisant des jeux sérieux, Peterson (2009) met en lumière la plus-value apportée par les jeux de simulation et les jeux de rôle. L'utilisation de la langue étrangère de manière pragmatique pour la résolution de problèmes semble ainsi favoriser la motivation de l'apprenant et le plaisir dans l'apprentissage.

Peterson souligne cependant l'utilisation de supports d'accompagnement adaptés aux compétences langagières de l'apprenant. Mandin (2011) préconise elle aussi la résolution de problèmes, mais également la possibilité donnée au joueur d'agir sur le déroulement de l'action (voir aussi Schmoll 2011).

L'action est aujourd'hui un élément central de l'enseignementapprentissage des langues étrangères. La perspective actionnelle, largement diffusée par le Cadre européen commun de réference pour les langues, envisage l'apprenant comme un acteur social ayant à accomplir des tâches dans des circonstances et un environnement donnés. Pour la réalisation de la tâche, l'apprenant a recours à des activités de com- 
Réalité virtuelle et jeux: de nouveaux outils pour des apprentissages plurilingues?

G. SChlemmingeR, M. Roy, M. Veit, A. Capobianco,

G. NOEPPEL

LA RÉALITÉ VIRTUELLE ET LE JEU AU SERVICE DE L'APPRENTISSAGE DES LANGUES munication langagières et au non verbal. Ainsi, la langue apprise constitue un outil au service de l'action et bénéficie de l'action comme support d'apprentissage. C'est sur cette approche holistique que se fonde la réflexion sur la Discipline Enseignée en Langue 2 (EMILE/CLIL) (Schlemminger 2008). Contrairement à la classe de langue classique, la langue apprise n'est plus l'objet principal d'étude, mais devient un outil fonctionnel pour des interactions centrées sur le contenu d'apprentissage et l'acquisition de nouveaux concepts. Ici, la focalisation sur les formes de la langue laisse place à la construction de savoirs adaptés au niveau des apprenants.

Voici deux exemples de logiciels de jeux sérieux pour l'apprentissage du FLE (français langue étrangère) (2):

Théleme, développé par Almédia (2009) avec l'appui du laboratoire «Cultures et sociétés en Europe» de l'Université de Strasbourg, est un jeu sérieux en ligne multi-participants. Une fois son avatar choisi, le joueur évolue dans un univers de cape et d'épée, se voyant confier des missions par les personnages du scénario. Sa réussite dans le jeu dépendra des actions entreprises et de sa capacité à communiquer en français. D'un point de vue technique, l'univers de jeu est représenté visuellement en 2D isométrique. Les dialogues ont lieu à l'écrit par l'intermédiaire d'un chat et le joueur déplace son personnage avec la souris de l'ordinateur. Développé à partir de 2007 et mis en ligne en 2009, ce jeu fait figure de précurseur dans le domaine des jeux pour l'apprentissage des langues. À ce titre, il est accueilli positivement par la presse spécialisée et le grand public.

La même équipe lance en 2012 Les Éonautes (Almédia 2012), jeu sérieux d'apprentissage du français langue étrangère. Les Éonautes s'adresse davantage à des apprenants de niveau A2. Ce niveau semble adapté à la participation de l'apprenant au développement de l'action. Contrairement à Thélème, Les Éonautes permet à l'enseignant de communiquer directement avec chaque apprenant, mais surtout d'intervenir sur le scénario en sélectionnant différentes missions que l'apprenant se verra confiées. Il peut ainsi effectuer des choix didactiques, afin de créer un parcours d'apprentissage personnalisé pour les apprenants. L'analyse didactique de ces dispositifs d'apprentissage met en lumière la forte orientation actionnelle et la pratique fonctionnelle de la langue induite par les univers de jeu.

Nous venons de présenter les enjeux technologiques, pédagogiques et didactiques à la fois de la réalité virtuelle et du jeu sérieux. Dans le cadre du projet franco-allemand EVEIL-3D (Schlemminger et al. 2012), nous sommes en train d'élaborer, développer et mettre en œuvre tous les éléments de la réalité virtuelle pour la mettre au service de l'apprentissage des langues étrangères. Nous proposons aux élèves de plonger dans un monde numérique en trois dimensions. Un 
Réalité virtuelle et jeux: de nouveaux outils pour des apprentissages plurilingues?

G. SChlemminger, M. Roy, M. Veit, A. Capobianco,

G. NOEPPEL jeu les place en situation d'immersion sensorielle et interactive dans le but de susciter une implication plus forte. Ils voyagent dans le temps et découvrent l'histoire de la Cathédrale de Strasbourg en participant à sa construction.

Grâce à la toute nouvelle combinaison de la reconnaissance des gestes et de la parole, les joueurs pourront parler, agir et interagir comme dans le monde réel. La musique, les bruitages renforcent encore le sentiment d'être présent dans la scène virtuelle. Nous partons de l'idée que ces facteurs favorisent l'apprentissage des langues, à condition que les élèves soient également sollicités cognitivement par du contenu (Schwienhorst 2009).

Nous élaborons un scénario global basé sur le principe d'une variante de jeu de rôle appelé «jeu en réalité alternée» (aussi appelé Alternate Reality Game). Les jeux de rôles classiques placent chaque joueur dans la peau d'un personnage différent de sa propre personne qui évolue dans un univers fictif. Pour ce projet, ce type de jeu présente l'inconvénient de devoir faire intervenir un maitre du jeu humain qui a pour objectif d'animer et de créer des obstacles dans l'univers de jeu.

À l'inverse, le «jeu en réalité alternée» repose principalement sur le maintien du flou entre les expériences perçues lors du jeu et hors du jeu. Il permet au joueur de s'approprier la trame globale du scénario en recevant de nouveaux éléments un par un, plutôt qu'à travers la narration d'un maitre de jeu. Le joueur incarne sa propre personne comme un des protagonistes principaux de lintrigue. Le professeur n'est pas exclu de ce type de scénario, puisqu'il peut y tenir le rôle d'accompagnateur et de facilitateur de la progression des joueurs, à travers la présentation des nouveaux éléments du scénario aux joueurs.

L'utilisation du dispositif technique constitue un point culminant du scénario global puisqu'il engendre une inversion du rapport du joueur avec le jeu: le joueur était habituellement immergé dans l'univers de jeu, ici c'est le jeu qui s'invite dans l'univers du joueur.

Les études didactique et technique ont montré le besoin de créer des moments de jeu en dehors de l'utilisation du dispositif technique afin de pouvoir développer le scénario et le contenu pédagogique. Ces phases ne se déroulent pas les unes après les autres: la phase d'enquête s'étend par exemple tout au long de l'aventure.

Phase 1: enquête

Cette phase est la base du «jeu à réalité alternée». Elle permet d'amorcer l'enquête qui s'étend tout au long de l'aventure. Le joueur incarne sa propre personne dans cet univers et est projeté dans l'univers de jeu en utilisant des outils qu'il utilise quotidiennement pour mener à bien son enquête: sites internet, réseaux sociaux, messageries. 
Réalité virtuelle et jeux: de nouveaux outils pour des apprentissages plurilingues?

G. SChlemminger, M. Roy, M. Veit, A. Capobianco,

G. NOEPPEL

\section{Phase 2: immersion}

Cette phase se focalise sur l'utilisation du dispositif technique. Elle est divisée en deux parties: une première pour l'étalonnage de la reconnaissance de la parole, et une seconde pour le dispositif technique dans son intégralité.

Phase 3: visite

Enfin, cette phase constitue la concrétisation de ce qu'a vécu le joueur au travers des deux premières phases. Les rôles et les univers s'inversent, puisque c'est désormais le joueur qui explore le lieu dans la réalité environnementale en quête d'indices à ramener dans l'univers de jeu. Le schéma suivant permet de résumer les différentes phases du jeu et leur découpage:

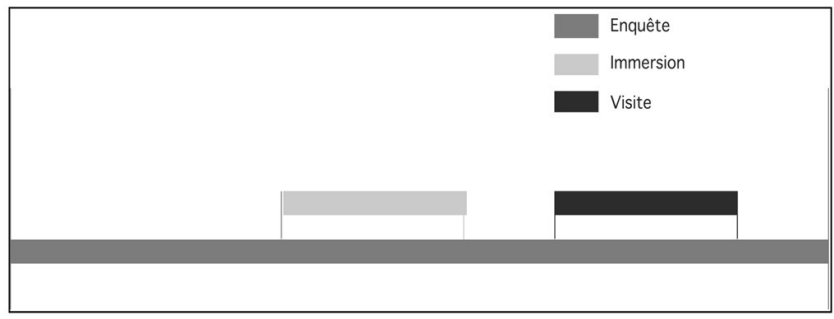

Fig. : Répartition des trois différents types de phases, la phase d'enquête englobe tout le jeu, les phases d'immersion et de visite interviennent aux débuts des second et troisième tiers de l'aventure

Avec le projet $E V E I L-3 D$, les élèves partent pour un voyage dans le temps: ils parcourent les 400 ans de construction de la cathédrale de Strasbourg. Ils ont pour mission de libérer Arthur Steinbach, tailleur de pierre bloqué dans le passé, enfermé dans les murs de l'édifice. À différentes époques de la construction et à plusieurs endroits de la cathédrale, les joueurs doivent, entre autres, reconnaitre des styles architecturaux ou comprendre la symbolique des couleurs de la rosace ou des statues d'animaux merveilleux. Au bout de leur voyage, ils graveront leur nom dans le mur de la tour, comme l'a fait Goethe. C'est ainsi qu'ils délivreront enfin le tailleur de pierre.

Le scénario gravite autour de deux personnes nommées Arthur et Céline Steinbach, respectivement père et fille. Le père est tailleur de pierre, alors que la fille est directrice d'une entreprise de recherche en réalité virtuelle 3D. Tous deux ont eu l'occasion de travailler sur le thème de la Cathédrale Notre-Dame de Strasbourg. Il y a peu, Céline a inventé une technologie révolutionnaire permettant à son utilisateur de visiter la Cathédrale 
Réalité virtuelle et jeux: de nouveaux outils pour des apprentissages plurilingues?

G. SChlemmingeR, M. Roy, M. Veit, A. Capobianco,

G. NOEPPEL

CONGLUSION: SOUTENIR LE PLURILINGUISME DANS

L'APPRENTISSAGE GUIDÉ DES LANGUES
Notre-Dame de Strasbourg à différentes époques du passé: cette technologie se nomme Eveil-3D. Arthur a tenu à être le premier à tenter l'expérience.

Malheureusement, au moment de la connexion d'Arthur, un virus informatique s'attaqua à Eveil-3D, condamnant l'accès au monde virtuel et empêchant Arthur de revenir. Céline assista impuissante à la disparition de son père et de toute la Cathédrale Notre-Dame de Strasbourg dans cet univers virtuel. Récemment, alors que tout semblait perdu, Céline reçut un message cryptique signé de son père: «Les architectes verront le chemin. Fais-leur confiance... Arthur». Quelques secondes plus tard, des dizaines d'établissements ont signalé avoir vu leur imprimante sortir la même feuille énigmatique...

Le projet EVEIL-3D est conçu pour le français et l'allemand langues étrangères. Malgré un travail de programmation didactique importante des dialogues, ce type de jeu peut être adapté à d'autres langues, cultures et contenus. D'ailleurs, la création d'un logiciel permettant aux enseignants de créer leurs propres scénarios est prévue. Une intégration didactique et pédagogique forte de la 3D immersive dans un scénario pédagogique soutenue par les TIC constitue un large éventail d'applications pour un apprentissage des langues centré sur les contenus. Cette démarche participe assurément à une forte motivation à apprendre les langues. La scénarisation, avec des phases avant et après le jeu constitue le défi majeur de l'intégration de la réalité virtuelle et de la 3D dans l'apprentissage.

Les objectifs pédagogiques pensés dès la conception du scénario ludique sont à priori mieux intégrés à l'univers de jeu, donc moins visibles pour le joueur. Cette «didactique invisible» (Ollivier 2010) présente une plus grande chance de motiver l'apprenant. Les objectifs sérieux sont utilisés en situation et deviennent plus fonctionnels puisqu'ils sont le médium qui permet d'avancer et de réussir dans le jeu. Si ces objectifs sont trop apparents, la notion de «didactique invisible» n'opère pas et n'a que peu d'impact sur l'implication du joueur-apprenant. Si, au contraire, l'objet parait trop ludique, l'apprenant risque de perdre de vue le contenu sérieux.

La technologie de réalité virtuelle constitue par conséquent un outil susceptible de favoriser l'implication de l'apprenant dans l'environnement d'apprentissage. L'immersion sensorielle et l'interaction qu'elle permet ne doivent cependant pas occulter le défi de scénarisation. L'enjeu est bien d'utiliser cette technologie 
Réalité virtuelle et jeux: de nouveaux outils pour des apprentissages plurilingues?

G. SChlemminger, M. Roy, M. Veit, A. Capobianco,

G. NOEPPEL

\section{BIBLIOGRAPHIE}

nouvelle en proposant une sollicitation cognitive adaptée à l'apprenant. Le jeu sérieux constitue une piste d'exploitation de la réalité virtuelle, faisant de la langue un outil fonctionnel alors utilisé par l'apprenant dans un environnement d'apprentissage adapté.

ALMÉDIA. 2009. Thélème. Strasbourg. http://www.theleme-lejeu.com ALMÉDIA. 2012. Les Éonautes.

Strasbourg. http://www.eonautes.com

ALVAREZ J. 2007. Du jeu vidéo au Serious game. Approches culturelle, pragmatique et formelle. Thèse de Doctorat en Science de la communication et de l'information. Toulouse: Université de Toulouse II et III. Site de la société Ja.Games:

http://ja.games.free.fr/These_SeriousGames/TheseSeriousGam es.pdf

AMOIA M., GARDENT G., PEREZ-BELTRACHINI, L. 2011. $A$ serious game for second language acquisition. Proceedings of the Third International Conference on Computer Aided Education (CSEDU 2011), Noordwijkerout, Pays Bas.

BLOCGI A. 2012. Univers virtuels : les jeunes comprennent très vite, Lokazionel.

http://www.lokazionel.fr/joomla15/index.php?option=com_con tent\&view $=$ article \&id=109: univers-virtuels-les-jeunes-comprennent-tres-vite \&catid=45:education \&Itemid $=125$

BOUVIER P. 2008. The five pillars of presence: Guidelines to reach presence, pp. 246-249 in Spagnolli A., International Society for Presence Research, et Annual International Workshop on Presence. 2008. Presence 2008: proceedings of the 11th International Workshop on Presence; Padova, Italy, 16-18 October 2008. Padova, CLEUP.

DJAOUTI D. 2011. Serious Game Design - Considérations théoriques et techniques sur la création de jeux vidéo à vocation utilitaire. Thèse de doctorat en informatique. Toulouse, Université de Toulouse III, Paul Sabatier.

FUCHS, P. et al. 2006. Le traité de la réalité virtuelle. Volume 1: L'homme et l'environnement virtuel, Paris, École des Mines.

HEERS R. 2005. 'Being There': Untersuchungen zum Wissenserwerb in Virtuellen Umgebungen. Tübingen: Eberhard-Karls-Universität Tübingen (thèse).

LANE H. C., HAYS, M. J., AUERBACH D., CORE, M. G. 2010. Investigating the Relationship between Presence and 
Réalité virtuelle et jeux: de nouveaux outils pour des apprentissages plurilingues?

G. SCHLEMmingeR, M. RoY, M. Veit, A. Capobianco,

G. NOEPPEL
Learning in a Serious Game. Intelligent Tutoring Systems, Vol. 6094, April, Springer, pp. 274-284.

MANDIN S. 2011. Jeux sérieux: quels apprentissages?, Agence des usages TICE, 14 janvier 2011. Site du CNDP: http: / /www.cndp.fr/agence-usages-tice / que-dit-larecherche/jeux-serieux-quels-apprentissages $\% \mathrm{C} 2 \% \mathrm{~A} 0-48 . \mathrm{htm}$ MCKERLICH R., RIIS M., ANDERSON T., EASTMAN B. 2011. Student Perceptions of Teaching Presence, Social Presence, and Cognitive Presence in a Virtual World, Fournal of Online Learning and Teaching, Vol. 7, No. 3, September.

MILGRAM P., KISHINO F. 1994. A taxonomy of mixed reality visual displays, IEICE Transactions on Information Systems, E77-D(12), pp. 1-15.

OLLIVIER G. 2010. Approche interactionnelle, tâches de la vie réelle et didactique invisible sur le web 2.0: pour plus d'authenticité et moins de simulation. Francparler, 'Faire Faire', avril. http://www.francparler.org/dossiers/fairefaire.htm

PATEL K., SANG H.-J., ROSEN D., RUZENA B., BAILENSON J.N. 2006. The Effects of Fully Immersive Virtual Reality on the Learning of Physical Tasks, pp. 87-94 in C.C. Bracken, M. Lombard, International Society for Presence Research Presence 2006: Proceedings of the 9th annual international workshop on presence, Cleveland.

PETERSON M. 2009. Computerized Games and Simulations in Computer-Assisted Language Learning: A Meta-Analysis of Research, Simulation \& Gaming, 41: 72-93.

RAGAN E.D., SOWNDARANJAN A. KOPPER R., BOWMAN D.A. 2010. The effects of higher levels of immersion on procedure memorization performance and implications for educational virtual environments, Presence: Teleoper. Virtual Environ. 19, 6: 527-543.

SANCHEZ-VIVES, M.V., SLATER M. 2005. From presence to consciousness through virtual reality, Nature Reviews Neuroscience, 6: 332-339.

SCHLEMMINGER G. 2008. Une approche didactique de l'enseignement bilingue: Le modèle rhénan, Synergies Pays germanophones $n^{\circ} 1$ : 97-111.

SCHLEMMINGER G. et al. 2012. EVEIL-3D. Environnements virtuels pour l'apprentissage. Lernen in virtellen Welten. http://www.eveil-3d.eu

SCHMOLL L. 2011. Usages éducatifs des jeux en ligne. L'exemple de l'apprentissage des langues, Jeux et Enjeux, Revue des Sciences Sociales n²4: 148-157.

SCHWIENHORST K.2009. Learning a Second Language in 
Réalité virtuelle et jeux: de nouveaux outils pour des apprentissages plurilingues?

G. SChlemminger, M. RoY, M. Veit, A. Capobianco,

G. NOEPPEL
Three Dimensions: Potential Benefits and the Evidence So Far, Themes in Science ans Technology Education, Vol 2, N 1-2, Special Issue: 153-163 (Klidarithmos Computer Books).

SLATER M. 2003. A note on presence terminology, Presence Connect 3, 3: 1-5.

\section{NOTES}

(1) Il s'agit d'un simulateur de régions 3D en code source libre, sous licence BSD, qui peut être visualisé par l'univers virtuel Second Life. Cet outil permet à l'enseignant de créer, de scripter et d'administrer son propre monde virtuel et aux joueurs-apprenants de chatter, d'importer des textures, modifier leur avatar et de terraformer (c'est-à-dire de transformer l'environnement pour le rendre «habitable» selon des conditions d'une vie de type terrestre) et, par l'intermédiaire d'avatars de participer à une ou plusieurs interactions plus ou moins guidées.

(2) Pour l'allemand, langue étrangère, le Goethe Institut propose un jeu sérieux pour apprendre l'allemand langue étrangère à partir du niveau A2: Lernabenteuer Deutsch - das Geheimnis der Himmelsscheibe. 